\title{
VE INFRASTRUCTURES REQUIREMENTS FOR COOPERATION AND KNOWLEDGE SHARING
}

\author{
Nuno Silva and João Rocha \\ Departamento de Engenharia Informática \\ Instituto Superior de Engenharia do Porto \\ Instituto Politécnico do Porto \\ Rua António Bernardino de Almeida \\ 4200-072 Porto - PORTUGAL \\ \{Nuno.Silva,Joao.Rocha\}@dei.isep.ipp.pt \\ www.dei.isep.ipp.pt/ nsilva
}

\begin{abstract}
Manufacturing systems are changing its structure and organization, focusing and limiting its core competencies. Proposed organizational and technological paradigms suggest dynamic organization forms like extended enterprise or virtual enterprises where entities outsource complementary products and actively pursuit cooperation with partners. However, a long way must be tracked in different domains and especially in organizational infrastructures and knowledge management. This paper characterizes and describes concepts and requirements related to the most common Distributed Manufacturing Systems approaches, emphasizing its needs about organizational and technological shifts. These ones are further interrelated with three organizational-technological approaches, which leads to the identification and description of needed functional services and particularly the service respecting to inter-domain knowledge operability.
\end{abstract}

\section{INTRODUCTION}

Manufacturing comprises all phases needed to provide a product or service from order booking through design, production, and marketing, and lately, recycling and final elimination. The majority of the manufacturing enterprises had the usual policy of directly controlling all the phases of business processes, within the enterprise using internal resources. However, different opinions (Agility Forum, 1997; NGM, 1997), suggest that multiple trends are affecting manufacturing context leading it to complete new challenges. Actually, all the referred trends express four distinct subjects (Sousa et al., 1999): (i) increased product variety over time, (ii) increased technological complexity, (iii) market globalization and (iv) increasing social pressures, which suggests different approaches, namely Adaptive and Responsive Information Systems; Knowledge Supply Chains; Extended Enterprise Collaboration and Enterprise Integration (Agility Forum, 1997).

A simple observation concerning technology support shows the enterprise had been typically characterized by monolithic structure, centralized co-ordination, low flexibility and adaptability, though highly efficient under intensive and repetitive processes (Sousa et al., 1999; Solberg and Kashyap, 1993). Hence, the formers technological concepts like Computer Integrated Manufacturing (CIM), lacks 
fundamental features like distribution, decentralization, flexibility, and adaptability (Silva and Ramos, 1999). With the emergence of large, distributed and decentralized systems, flexible and very dynamic, which the Internet is the most paradigmatic one, advantages of such features seems indubitable.

Even if major progresses are crucial to accomplish many of the requirements already experimented, these concepts look the correct approach. The question in most cases is the capacity to integrate disparate type approaches, architectures, technology and above all, the understanding about each particular domain. New forms of inter-operability must be achieved prior to any new significant evolution in the distributed manufacturing area or related ones.

The rest of the paper is divided in the following subjects. In Section 2, the most common DMS characteristics and behaviors are described. Next, in section 3, three organizational-technological paradigms are summarized and its conceptual operation compared, which allows to analyze and then to conclude the need for several basic but fundamental operational services. In chapter 4 , these services are conceptually and operationally described and finally in chapter 5, a general overview is presented and further potential directions are pointed out.

\section{DISTRIBUTED MANUFACTURING SYSTEMS}

Manufacturing systems are changing its structure and organization, evolving to more dynamic organization forms like extended enterprise or virtual enterprise (VE). A $\mathrm{VE}$ is a set of enterprises opportunely joined in logical and operational, although momentary manufacturing structure, co-operating to accomplish predefined and mutually accepted goals. Such enterprises comprehend multiples autonomous entities, each potentially composed by other autonomous inter-related co-operative enterprises, behaving globally as single one, through advanced communication supports, thus forming a cybernetic structure (Sihn, 1997). This kind of enterprises operate in a very dynamic, varying and challenging environment, where a new market opportunity, be a product or service (product for short), triggers the creation of a complete new logical enterprise structure, focused in providing the market with such new product.

Organizational concepts like Supply Chain, Extended Enterprise or Virtual Enterprise suggests the idea of Distribution, Decentralization, Dynamism, Autonomy; Ill-specification, Agility (Sousa et al., 1999). During operation these enterprises need important improvements, specially complementing (i) Reactivity with Awareness and Partnership, (ii) Flexibility and Adaptability with Co-operation, (iii) Autonomy with Agility. In order to accomplish these challenges, a transversal requirement observed is the understanding between different, autonomous, and distinct domain enterprises (Camarinha-Matos and Afsarmanesh, 1997). Usually, such enterprises have domain specific structures, operate proprietary/domainspecific systems, using (speaking) domain specific terms and expressions. In order to co-operate and co-ordinate activities between such enterprises, one must publicly share mechanisms to "reason" about its domain. It is important enterprises realize the need for knowledge management in opposite to information management and start operate considering knowledge the most important resource in the enterprise and for inter-operability. One of such mechanism is a shared ontology (Schlenoff $e t$ 
al., 1999; Ontology.Org, 1999). In Uschold et al., 1998; Gruninger and Fox, 1995, the major motivations for shared ontology exploitation are the enterprise modeling and inter-operability in the enterprise either be a monolithic or distributed one. Hence, knowledge management in general and shared ontology's in particular, perfectly fits the requirements for inter-enterprise knowledge management.

\section{ORGANISATIONAL-TECHNOLOGICAL PARADIGMS}

However, such concepts are too vague and its interpretation varies from implementation to implementation. New organizational concepts have been proposed to prevent misunderstandings and help in implementing such systems. Three of these paradigms are:

- The Fractal Factory (FF) paradigm is based on mathematical fractal concept and its associated theory of chaos, These systems are characterized by constant evolution between partners, environment and internal operation, abandoning the function-oriented organization and adopting a project-oriented organization (Sihn, 1997). This process is very dynamic (and presumable successful) since the resource can (well) decide about its own behavior and does not rely on a higher entity to do it. Thus, the fractal factory must be understood as a non-linear system (Sihn, 1997), structurally reactive and adaptive to the dynamic context. In addition, the concept easily models different enterprise dimension, like strategic, social, cultural, informational and technological (Zelm et al., 1995);

- The Bionic Manufacturing System (BMS) is based on structures and behaviors observed in biology (Tharumarajah et al., 1996). From the simplest through the most complex living form, inside of certain hierarchically ordered relations, all manifest autonomy, spontaneous behavior, social harmony, consistency and goal orientation. The self-division mechanism based in the genetic code (DNA) information, is the main responsible for this behavior. BMS tries to define a parallelism between biological systems and manufacturing systems, traducing the DNA inheritance in biology to characteristics, states, behavior and domain knowledge in manufacturing;

- The Holonic Manufacturing Systems (HMS) arises from Herbert Simon and Arthur Koestler studies about biological society evolution and organization. Koestler perceived that Simon's analyzed intermediate forms are simultaneously a part and a whole, a container and a contained, a controller and a controlled. In order to designate these hybrid nature and behavioral entities, (Koestler, 1967) proposed the term Holon as the combination of the Greek word Holos (whole), and the on suffix (particle, like in neutron). Each holon combines its set of competencies with its lateral partners, with whom co-operate in order to achieve both individual and system goals. This suggests the holon is an autonomous entity, including operational features and individual goals. System goals and plans are partially defined in higher holons and travelling down the holarchy, they are progressively refined. The result is a highly complex but reactive system with minimal concession to efficiency. 
These paradigms suggest the idea that manufacturing systems, despite the increased autonomy required by individual entities, it will continue to need a hierarchical structure in order to maintain the overall system coherence and objectivity, and guarantee the inter-entities conflict resolution arising from the individual and autonomous attitude of the entities. The three paradigms have different origins, which imply different technical approaches. The BMS paradigm suggests some properties intimately similar to modern enterprises, specially relating to the environment, full of information and chances to improve business. On the other hand, the Fractal Factory paradigm is the most modern approach since it relies on individual entity autonomy and vitality to overcome responsiveness and performance. Further, it is based on mathematical formalisms that make it the preferred approach to design and specification. However, its application tends to be complex especially respecting to implementing navigation and co-ordination mechanisms. Finally, the HMS paradigm, being the most traditional due to its structure and organization, it is easily adaptable to the Object Oriented paradigm, thus sub-specification and specialization. Also, is the most stable due to the hierarchical rules statically defined, the canon.

These paradigms were proposed in order to provide the enterprise with standard and effective organization and technological approaches, which does not mean that hybrid approach or even complete different ones, could not be developed or combined.

\section{FUNCTIONAL SERVICES}

A long distance must be tracked from the conceptual model through the complete realization of these systems, especially in organizational infrastructures and knowledge management.

Organizational infrastructures requirements highly rely on the employed organizational paradigm, but Communication, Security, Coherence, Reliability and Domain facilities can by systematized and several procedural patterns were identified. Considering it, four complementary services have been recognized: Identification, Information, Pooling, and Domain services.

The Information Service, (InfSys) is responsible for inclusion and exclusion of any entity in the system community. To adhere, each entity should request it. The service involves four phases:

- Identification. If the entity is not recognized in the system or if it is already registered, then the request is refused;

- Authentication. This phase guarantee the entity is whom it claims to be. At the moment, it comprehend a bilateral authentication process based on public/private key mechanism;

- Registration. This phase correspond to the insertion of the entity identification in the InsSys database and consequently the entity full admission to the system. From now on, the entity can contact any other member and vice-versa;

- Unregistration is the Registration opposite phase. The entity is excluded from the system, thus it is no longer allowed to contact other member and vice-versa. 
Figure 1 presents the conversations required completing the first three phases.

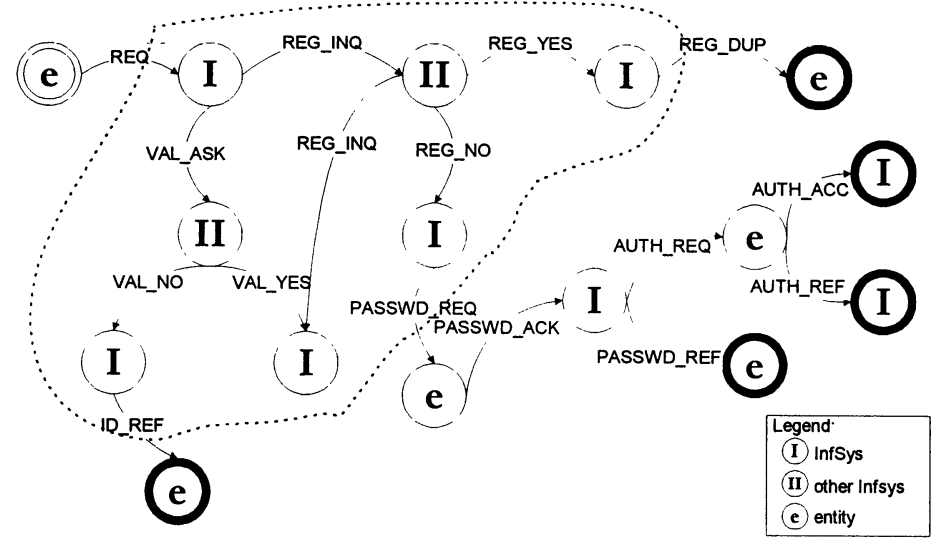

Figure 1 - Identification Service conversation through Registration

Additionally, the InfSys service is also responsible for Directory Service, which provides the system society with domain public information, namely with capabilities one wants advertise to the community (e.g. performing operations, features, reliability, costs). To address reliability, multiples InfSys entities are allowed, even if incoherence easily occurs in such conditions. To prevent these problems, co-operation and publishing rules are essential and should be strictly followed: (i) Information has a single owner; (ii) The owner is responsible for information management (publish, update and unpublish); (iii) Information owner limits information time validity; (iv) Information can not be updated until corresponding supplied information looses validity; (v) Only InfSys provides published information; (vi) Information is valid exclusively during owner InfSys registration; and (vi) When queried about unfamiliar information, InfSys query its InfSys partners for it, thus, acting as a broker.

Combining Identification Service and Directory Service in an integrated system, information coherence and validity are easier to maintaining since it is assured an unique connection to each registered entity. That way, any entity can not duplicate or publish opposite information.

The Pooling Service (PoolSys) concerns with message dispatching to entities temporarily unreachable or unregistered in InfSys. The PoolSys entity stores and delivers the requested message as soon as the destination entity is reachable. Each entity providing the service has to register and further publish its capability in InfSys as any other capability. In order to achieve reliability (even it is not frequently demanded), multiple PoolSys entities are allowed in the system even no co-ordination or co-operation is required. Any message is stored in PoolSys entity database without any processing. PoolSys entity periodically queries its associated InfSys entity for the destination entities and when available, dispatch the message.

Fractal and Holonic paradigms propose the regrouping property based on different perspectives and reasons, during the system operation. These sets of entities grouped together are named domain. Dynamic grouping impose several constraints in system management, namely in inter-domain relation and information management, which suggests the pertinence of an additional service responsible for 
domain boundaries and inter-domains relations. This service would be responsible for logically group multiple entities, behaving globally as hierarchical systems and proving and interface with its lateral partners. The service should filter and route conversations messages received from and sent out domain boundaries. Such service is named Domain Service (DomainSys).

Each DomainSys entity represents in a wider context, a set of entities acting as unique, autonomous, self-regulated entity, including public capabilities and constraints, behaviors and plans. Internally, each domain is a completely independent identification and information context, applying previously described restrictions and functional requirements (Figure 2).

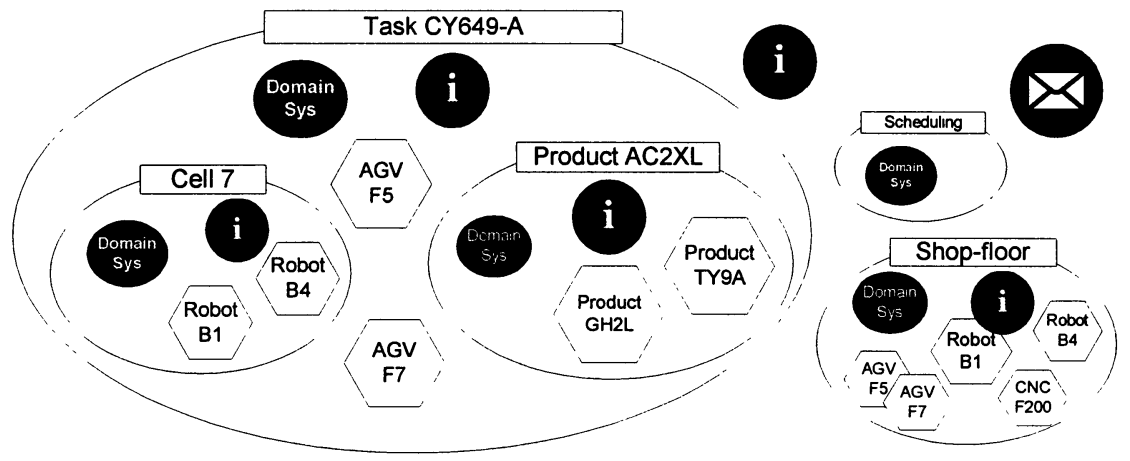

Figure 2 - Domain Service representation

The represented DomainSys entity (based in the "virtual class" DomainSys), is the domain specific implementation of behaviors, plans, capabilities, processes and flow inside the domain. The domain internal InfSys, respects to its specific context and provides the mechanisms to share information inside the domain. Additionally each entity taking part of multiple domains is able to publish specific domain public information in other domains, what allows a wide sharing mechanism.

As conceived, Domain Service (DomainSys) allows defining domains in the system, either at developing time or as emergent structures. Any of these services are supplied as predefined entities and independent development layers. Additionally, providing both server and client support, task specific entities are able to both provide and require these services. Figure 3 represents this approach.

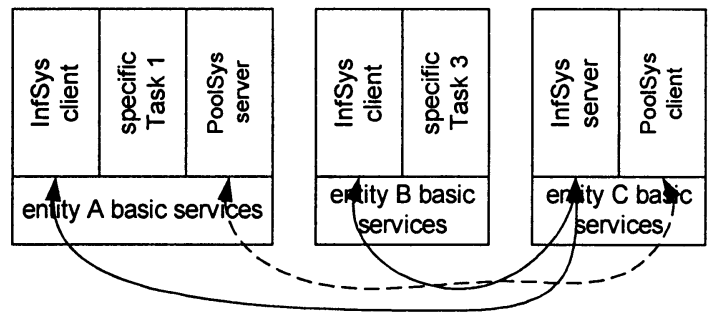

Figure 3 - Functional Services Implementation

In addition, Fractal and Bionic approaches suggest a very high co-operation between a very dynamic set of entities. Thus, each entity may contacts heterogeneous ones, which conversations and related knowledge are potentially 
distinct. A service that would negotiate these issues without or minimal user intervention would be a huge improvement during inter-domain/enterprise co-operation. Such service should negotiate conversation languages, formats and protocols, and specially, negotiate and automatically construct a shared (accepted) ontology. Some agent communication language as FIPA ACL provides some primitives helping conversation setup, namely conversation language and information format negotiation.

Consider the more common ontology architectures, which are composed by multiples layers, each inheriting and specializing multiple lower ones (Uschold et al., 1998), creating even more detailed one. Figure 4 exemplifies this method, in two different ontologies.
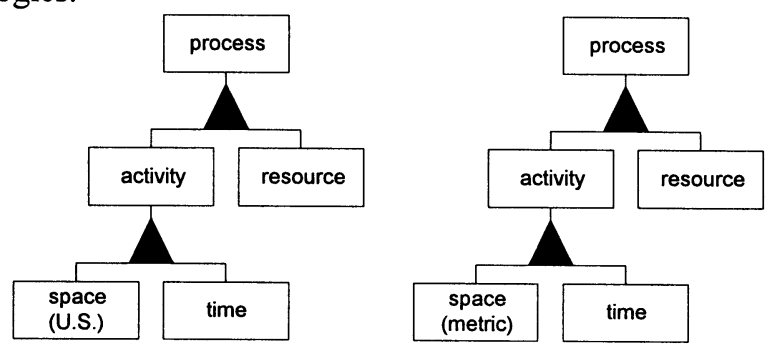

Figure 4 - Inheriting and specialising ontologies.

Observing both ontologies, some similar elements are identified (space-metric and space U.S.) while others are potentially distinct. The procedure attempting to combine both ontologies should, in a primary step start to combine lower layer ontologies, minimizing higher incompatibilities, i.e., resolve incompatibilities as they begin. In many cases, it should not be possible to come up with an agreement that will impose different approaches.

The most usual method is the mapping concepts between ontologies. In the example of Figure 4, if no agreement on choosing a single ontology, a mapping function must be applied to any translated space object, in order to each entity reasons about correct values. However, even if ontologies are increasingly implemented considering the mapping features, circumstances where the method is applicable are still a few or the do not fulfill the entire conversation and knowledge passing. The suggested and more advanced intelligent approach is the combination of reasoning procedures on ontology contents, which allows the creation and discovery of corresponding/mapping points between ontologies, and the application of agreement mechanisms.

In a pragmatic perspective, the first approach is conceivable in a homogeneous or (at least) stabilized context like the extended enterprise. In contrast, if the organization structure is looser (i.e. VE) or very distinct technological levels occurs (typically in Supply Chain based organizations), a more dynamic and knowledge based solutions should be considered.

\section{SUMMARY AND FURTHER DIRECTIONS}

This paper discusses technological support of an emergent organizational paradigm: the Virtual Enterprise and some derivative forms. 
The holonic, bionic and fractal approaches were briefly described and compared which allowed finding common characteristics and behavior patterns and in some sense, advocate these paradigms are not always contradictory and can even be combined into specific systems. Considering the identified patterns, it was considered necessary to provide the system with specific services concerning Identification, Information, Pooling and Domain services. In addition, a brief analysis about inter-domain knowledge sharing demonstrated the need to improve functional services, in this particular to the automatic construction of shared and accepted ontologies.

Considering the conceptual behavior characteristics of the analyzed paradigms, namely the dynamic search and consequent grouping in task specific domains, the negotiation service is considered a very promising improvement vector.

Some implementations and experiences with shared auto-configured ontologies based in ontology reasoning and agreement mechanisms are carrying on in our research group, although in a very crude approach.

\section{REFERENCES}

1. Agility Forum; http://www.agilityforum.com; 1997.

2. Camarinha-Matos L.M., Afsarmanesh H.; Virtual Enterprises: Life cycle supporting tools and technologies; in Handbook of Life Cycle Engineering: Concepts, Tools and Techniques, A. Molina, J. Sanchez, A. Kusiak (Eds.), Chapman and Hall, 1997.

3. Gruninger M. and Fox M.S.; Methodology for the Design and Evaluation of Ontologies; (1995); Workshop on Basic Ontological Issues in Knowledge Sharing, IJCAI-95, Montreal; 1995.

4. Koestler A.; The Ghost in the Machine; Hutchinson \& Co, London, 1967.

5. NGM (Next-Generation Manufacturing); A Framework for Action; Executive Overview; January 1997; URL: http://www.dp.doe.gov/ngm/ngm.pdf; 1997.

6. Ontology.Org; The need for a shared ontology; http://www.ontology.org/main/pagel .html; 1999.

7. Schlenoff Craig, Ivester Rob, Knutilla Amy; A robust process ontology for mfg sys integration; National Institute of Standards and Technology Gaithersburg, MD 20899; URL: http://www.ontology.org/main/papers/psl.html; 1999.

8. Sihn Wilfried; The Fractal Factory: A Practical Approach to Agility in Manufacturing; Proceedings of The Second World Congress On Intelligent Manufacturing Processes \& Systems, pp. 617-621, Budapest, Hungary, June 10-13, 1997.

9. Silva N. and Ramos C.; Proposal for Inter-Enterprises Negotiation Infra-Structures using an Holonic approach; Proceedings of 1st International IFAC Workshop on Multi-Agent Systems in Production; Wien, Austria; 2-4 de December 1999.

10. Solberg J. and Kashyap R.; Research in Intelligent Manufacturing Systems; Proceedings of the IEEE, vol. 81, nº 1 , January 1993.

11. Sousa P., Silva N., Heikkila T., Kollingbaum M, Valckenaers P.;Aspects of Co-operation in Distributed Manufacturing Systems; Proceedings of the 2nd International Workshop on Intelligent Manufacturing Systems (IMS-Europe'99); Leuven, Belgium; 22-24 Setembro de 1999.

12. Tharumarajah A., Welles A.J. and Nemes L.; Comparison of the bionic, fractal and holonic manufacturing system concepts; International Journal of Computer Integrated Manufacturing, 1996, vol. 9, no 3, 217-226.

13. Uschold M., King M., Moralee S. and Zorgios Y.; The Enterprise Ontology; The Knowledge Engineering Review, Vol. 13, Special Issue on Putting Ontologies to Use; Eds. Mike Uschold and Austin Tate; 1998.

14.Zelm M., Vernadat F.B. and Kosanke K.; The CIMOSA business modeling process; Computers in Industry 27, pp. 123-142; 1995. 